\title{
Variations of the velocity field of the solar atmosphere according to observations $\mathrm{SOHO} / \mathrm{MDI}$ and NSO/KPVT
}

\author{
A.G.Tlatov and V.V. Vasil'eva \\ Kislovodsk Solar Station of the Pulkovo Observatory, Russia, email: solar@narzan.ru
}

\begin{abstract}
According to daily observation SOHO/MDI during 1996-2003 and NSO/KP Vacuum Telescope during 1978-2003 magnetic elements with intensity of the magnetic field above background have been allocated. The knots were then identified that allowed to trace their displacement comparatively of heliographic grid. Thus, fields of speeds for the period with 1978 on 2003 have been constructed. Variations of speed meridional circulation are found out. The basic direction of speed meridional drift is directed to poles. At the same time it is shown, that during a maximum of activity at latitude less than $20^{\circ}$ arise currents in the direction of equator. Streams in the direction to equator arise and during a minimum of activity at latitude about $40-60^{\circ}$. Fields of speeds on various phases of solar cycle are constructed.
\end{abstract}

In this analysis we were used observations of magnetic fields MDI/SOHO and KPVT. The technique of allocation of magnetic structures was following. On each image pixels with intensity of magnetic field on absolute value not less $8 \mathrm{Gs}$ for MDI and 10Gs for Kitt Peak were allocated. Groups of the allocated pixels could form the structures having the common border, separating structure from background magnetic field. Were taken into account elements having the area not less than 20 million parts of hemisphere (mph). For determination of the horizontal velocity was identification of such magnetic structures on the next images is carried out. Thus, the identification was conducted at minimization of deviations from expected position.

For checking the methods of allocation tracers and its applicability for definition of speeds the comparative analysis of definition of speed of rotation is carried out. For elements of the greater area the law of differential rotation differed from it. Formulas of approximation for elements of the various sizes are resulted in Table 1. For elements of the small size the formula of approximation is close to values received in article Komm, Howard \& Harvey (1993).

In figures 1 and 2 latitude-time diagrams of speed meridional circulation are resulted according to observations Kitt Peak and SOHO/MDI. The latitude-time distribution of meridional circulation determined in the present work shows, that the field of velocities considerably depends on a phase of a cycle (Snodgrass \& Diley (1996)). With development of activity in at equatorial areas at latitudes is lower $20^{\circ}$ current it is directed to equator. The line section meridional streams of various directions lays near to the center of the butterfly and moves to equator. Probably, it occurs because from an emerging magnetic stream in the field of occurrence of spots there are horizontal currents in the opposite sides. In a minimum of activity meridional drift in each hemisphere basically is directed to poles, except for area of latitudes $40-60^{\circ}$ where it is directed to equator. Probably, variations meridional velocities at these latitudes also are caused by emersion of a stream in the top layers of a solar atmosphere. 


$\begin{array}{lr}\text { Area }(m p h) & \text { approximation deg/day } \\ 80-100 & 13.42-1.97 \cdot \sin ^{2}(\phi)-1.87 \cdot \sin ^{4}(\phi) \\ 100-300 & 13.38-1.93 \cdot \sin ^{2}(\phi)-1.96 \cdot \sin ^{4}(\phi) \\ 500-1000 & 13.34-2.03 \cdot \sin ^{2}(\phi)-2.15 \cdot \sin ^{4}(\phi) \\ 1000-2000 & 13.24-2.14 \cdot \sin ^{2}(\phi) \\ 3000-5000 & 13.15-2.27 \cdot \sin ^{2}(\phi) \\ >5000 & 13.05-2.33 \cdot \sin ^{2}(\phi)\end{array}$

Table 1. Formulas of approximation of differential rotation for elements of the various sizes
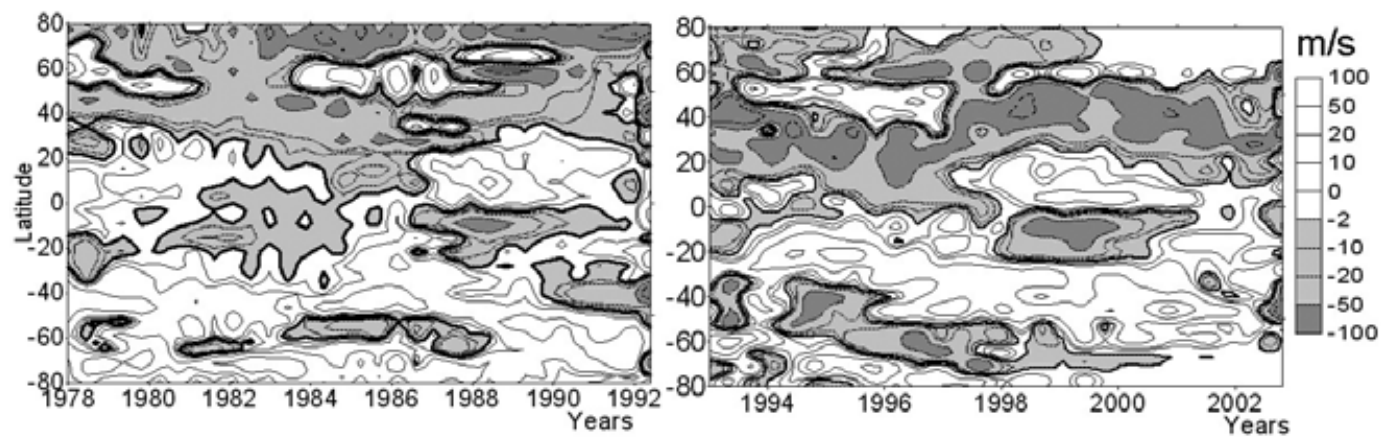

Figure 1. Distribution of drift meridional circulation on data KPVT.

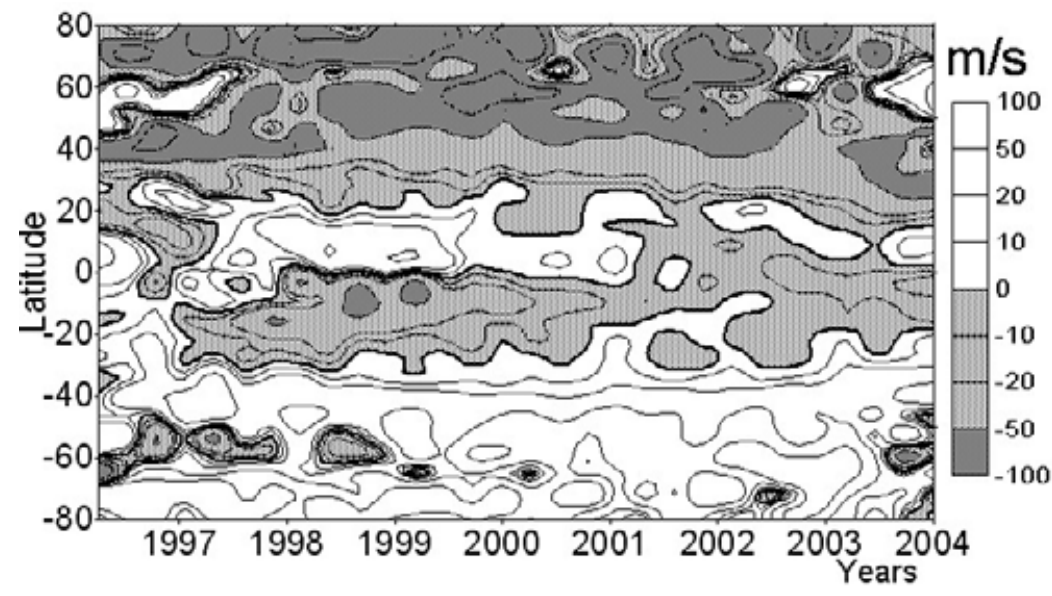

Figure 2. The latitude time diagram of distribution meridional circulation drift determined according of MDI data. The numbers element for calculations has formed 100000 . The dark color allocates the elements moving to North Pole, white - to Southern pole.

\section{References}

Komm, R.W., Howard, R. \& Harvey, J.W. 1993 Solar Phys., 143, p.19.

Snodgrass, H. \& Diley, S.B. 1996 Solar.Phys., 163,p.21. 